\title{
Panorama geral sobre dados hidrológicos com ênfase em eventos hidrológicos extremos
}

\author{
General overview of hydrological data with emphasis in \\ extreme hydrological events
}

\begin{abstract}
Franciele Maria Vanelli ${ }^{1}$, Fernando Mainardi Fan ${ }^{1}$ (D), Masato Kobiyama ${ }^{1}$ (D)
${ }^{1}$ Universidade Federal do Rio Grande do Sul - UFRGS, Porto Alegre, RS, Brasil. E-mails: franciele.vanelli@ufrgs.br, fernando.fan@ufrgs.br, masato.kobiyama@ufrgs.br
\end{abstract}

\begin{abstract}
Como citar: Vanelli, F. M., Fan, F. M., \& Kobiyama, M. (2020). Panorama geral sobre dados hidrológicos com ênfase em eventos
\end{abstract} hidrológicos extremos. Revista de Gestão de Água da América Latina, 17, e24. https://doi.org/10.21168/rega.v17e24

\begin{abstract}
RESUMO: 0 conhecimento depende da existência de dados. A hidrologia moderna tem sido, prioritariamente, pautada em dados quantitativos provenientes das estações de monitoramento. No entanto, outras fontes podem fornecer dados relevantes ao avanço da hidrologia como demonstrado por pesquisadores desde a década de 1970. 0 panorama geral sobre o assunto evidencia a falta de padronização das terminologias dos tipos de dados e de fontes, podendo conduzir a equívocos. Além disso, ainda não existe consenso sobre o uso de dados provenientes de outras fontes, além das estações de monitoramento. Neste contexto, o presente estudo sugere a padronização nas denominações dos dados, bem como em suas fontes. Para isso, levou-se em consideração as características do registro, classificando-os em: dados sistemáticos e dados não sistemáticos. Os dados sistemáticos são dados quantitativos, mensurados continuamente ao longo do tempo com intervalo temporal pré-definido em um determinado local, enquanto os dados não sistemáticos são provenientes de uma grande variedade de outras fontes de dados que se caracterizam, principalmente, por registros cuja posição e intervalo temporal são variáveis. Quanto às fontes dos dados, sugere-se a classificação de acordo com a sua origem: evidência instrumental evidência natural e evidência documental. Considerando a relevância dos dados não sistemáticos, principalmente ao que tange eventos hidrológicos extremos, sugere-se o uso integrado de dados sistemáticos e não sistemáticos aplicando o método de triangulação de dados.
\end{abstract}

Palavras-chave: Dados Sistemáticos; Dados Não Sistemáticos; Comunidade; Desastres; Reconstrução; Sociohidrologia.

\begin{abstract}
Knowledge depends on the existence of data. Modern hydrology has been primarily based on quantitative data from gauging stations. However, other sources may provide data relevant to hydrology progress as demonstrated by researchers since the 1970s. The general picture on the subject highlights the lack of standard terminologies for data types and sources, which can lead to misunderstandings. Besides, there is still no consensus on the use of data from sources other than monitoring stations. In this context, in the present study, we suggested standard denomination for data and their sources. We classified from the recorded characteristics as systematic data and nonsystematic data. Systematic data are quantitative data, measured continuously over time with a pre-defined time interval at a given position, while non-systematic data are from a wide variety of other data sources, whose position and time interval of data are variable. We also classified the data sources according to the origin: instrumental evidence, natural evidence, and documentary evidence. Considering the relevance of non-systematic data, mainly concerning extreme hydrological events, we suggested the use of both data - systematic and non-systematic - applying the data triangulation method.
\end{abstract}

Keywords: Systematic Data; Non-systematic Data; Community; Disasters; Reconstruction; Socio-hydrology.

\section{INTRODUÇÃO}

Os dados são ponto de partida para entender melhor o mundo (Herzog, 2015). Conforme Rowley (2007), a hierarquia dado - informação - conhecimento - sabedoria (hierarquia DICS, em inglês datainformation-knowledge-wisdom (DIKW) hierarchy) é, em geral, mencionada implicitamente ao

Recebido: Outubro 05, 2020. Revisado: Novembro 19, 2020. Aceito: Dezembro 04, 2020. 
assumir que dados podem gerar informações, que as informações podem gerar conhecimento e conhecimento pode gerar sabedoria. Assim, em suma, o avanço em cada ciência depende de dados obtidos em diferentes fontes de acordo com o objeto de estudo.

Na hidrologia, Robert Horton, conhecido como pai da hidrologia moderna (Pennington \& Cech, 2010), escreveu em 1931 que a hidrologia, assim como a geografia e a meteorologia, se desenvolvia principalmente em caráter qualitativo, emergindo como uma nova ciência a partir do seu desenvolvimento quantitativo (Horton, 1931). A aplicação de métodos estatísticos na hidrologia a partir da publicação do livro "Flood Flows" por Hazen em 1930 (Dooge, 2004), assim como as instalações de estações de monitoramento por serviços hidrológicos nacionais de diversos países a partir do início do século XX (Brázdil \& Kundzewicz, 2006) corroboram com a afirmação de Horton. Portanto, apesar das primeiras referências de medição do nível de água datarem de 3.000 a.C. no rio Nilo (Dooge, 2004) e da publicação de Pierre Perrault sobre a relação precipitação-vazão em 1674 (Nace, 1969), foi somente a partir do início do século XX que a mensuração das variáveis hidrológicas continuamente pelas estações de monitoramento e o uso de métodos estatísticos se tornaram base da hidrologia.

Entretanto, apenas uma pequena parcela das bacias no mundo é monitorada, repercutindo em uma limitação temporal e espacial dos dados (Kidd et al., 2016). Ao tratar de eventos hidrológicos extremos, as próprias características dos eventos repercutem na limitação ou baixa qualidade dos dados oriundos de estações de monitoramento. Os eventos hidrológicos extremos são eventos associados à abundância ou escassez da água e ocorrem com maior magnitude e menor frequência. Assim, são eventos com poucos registros ao longo do tempo, com incremento nas incertezas associadas ao equipamento de medição ou no método de estimativa aplicado, como no caso da extrapolação da curva-chave, tanto para mínimas quanto para máximas (McMillan et al., 2018). Além disso, em eventos de máxima, o fluxo violento da água pode ser perigoso ao operador e/ou aos equipamentos causando danos parciais ou totais (Brázdil et al., 2006).

Reconhecendo as limitações dos dados provenientes das estações de monitoramento, principalmente quanto aos eventos hidrológicos extremos, outras fontes de dados podem fornecer informações e, consequentemente, conhecimento (hierarquia DICS). Conforme Francés et al. (1994), a partir da década de 1970, diversos autores reconheceram o potencial de outras fontes de dados para análise de frequência de inundações, como por exemplo, marcas de cheia, registros em jornais e livros, comunicação oral, evidências botânicas, dentre outros. Essas fontes fornecem dados qualitativos que possibilitam inferências quantitativas de modo que o uso conjunto com os dados das estações de monitoramento possibilita uma melhora no nível de confiança na estimativa do perigo associado a eventos hidrológicos extremos (Fernandes et al., 2013; Barriendos et al., 2014).

Os avanços tecnológicos do final do século XX e início do século XXI possibilitam novas fontes de dados, que podem auxiliar em estudos hidrológicos desde o registro dos dados até o processamento. Desde a disponibilidade de computadores e celulares com maior capacidade de processamento e internet até o desenvolvimento do sensoriamento remoto. Nas décadas recentes, os registros podem ser realizados pelas câmeras dos celulares, veículos aéreos não tripulados (VANTs), mídias sociais, dentre outros. Assim, existe uma grande quantidade de dados que podem fornecer informações úteis à hidrologia. Além disso, a aplicação de algumas técnicas estatísticas, como por exemplo redes neurais, podem auxiliar na busca de padrões em grandes conjuntos de dados (big data).

Os registros dos eventos passados marcam o início das pesquisas sobre enxurradas, pois os pesquisadores, independente do objetivo, iniciam suas investigações a partir de dados dos eventos passados (Kaiser et al., 2020). Essa afirmação pode ser estendida aos demais eventos hidrológicos. De acordo com Montanari \& Koutsoyiannis (2014), os dados e, portanto, a observação do passado, são a chave para alcançar uma melhor compreensão dos processos hidrológicos e das mudanças ambientais.

Sob a ótica que sem dados não há o desenvolvimento de novos conhecimentos, discute-se sobre o valor relativo dos dados provenientes de estações de monitoramento e dos dados de fontes distintas. Blöschl et al. (2019) apresentaram os 23 principais problemas atuais da hidrologia e elencaram o uso de dados de fontes distintas como o 17ํㅜ problema. Em virtude da maior oferta e variedade de dados, Montanari \& Koutsoyiannis (2014) destacaram a integração de dados de diferentes fontes como uma das prioridades aos hidrólogos. No entanto, para responder esses questionamentos é necessário compreender o que são dados, sua classificação e suas fontes.

Nessa perspectiva, o presente estudo fornece um panorama geral sobre os dados na hidrologia, sugerindo uma denominação padrão de acordo com as características de registro e padronização das terminologias das fontes com base em sua origem. Por fim, destaca-se o uso integrado de dados 
provenientes de diferentes fontes e são feitas reflexões e propostas de como pode ser ampliada a disponibilidade de dados.

\section{CLASSIFICAÇÃO DOS DADOS}

Os dados podem ser classificados de acordo com muitos critérios que podem variar em cada ciência. No entanto, uma das principais classificações dos dados é como quantitativo ou qualitativo. Os dados quantitativos são provenientes de mensuração e representados por números cuja análise é baseada em métodos estatísticos, enquanto que os dados qualitativos se referem a descrições que podem estar no formato de textos, imagens, peças audiovisuais, documentos, objetos pessoais, dentre outros (Sampieri et al., 2013).

No escopo da hidrologia, pesquisadores como Francés et al. (1994), Benito et al. (2004), Brázdil et al. (2006) empregaram a denominação: dados sistemáticos e dados não-sistemáticos. Conforme esses autores, os dados sistemáticos são registrados sequencialmente em um intervalo de tempo pré-definido auxiliados, direta ou indiretamente, por equipamentos associados à hidrologia. Em geral, dados sistemáticos se referem aos dados provenientes das estações de monitoramento. Já os dados não sistemáticos contemplam as evidências identificadas no ambiente natural e os dados provenientes de registros históricos ou observações humanas, cujos registros são pontuais no tempo e/ou no espaço. A natureza dos dados não sistemáticos enfatiza o fenômeno ocorrido no passado e a sua compreensão.

Seibert \& McDonnell (2002) introduziram o conceito de soft data e hard data na hidrologia. 0 termo hard data se refere a dados concretos remetendo às séries temporais de dados mensurados (Seibert \& McDonnell, 2015; Arnold et al., 2015). Em contrapartida, soft data se refere a dados frequentemente irregulares, descontínuos e numericamente aproximados (Seibert \& McDonnell, 2002). Em Merriam-Webster Dictionary (Merriam-Webster, 2020), esse termo pode ser traduzido como dados interpretativos. Esses dados podem gerar incertezas devido à subjetividade, entretanto Seibert \& McDonnell (2002) salientam que é melhor usar esses dados do que negligenciar esse conhecimento e apenas empregar dados mensurados para calibração do modelo hidrológico. Seibert \& McDonnell (2015) demonstraram que à medida que os dados mensurados são mais limitados, os dados interpretativos tornam-se mais úteis, denotando a importância do diálogo entre o diagnóstico de campo e a modelagem. Neste contexto, conforme Seibert \& McDonnell (2002), os dados interpretativos podem ser usados para avaliar aspectos das simulações do modelo para os quais não existem dados concretos disponíveis e avaliar se os valores dos parâmetros são razoáveis com base na experiência de campo.

Neste contexto, evidencia-se que os autores utilizam diferentes terminologias para se referir aos dados das séries temporais mensurados: dados sistemáticos (e.g. Francés et al., 1994; Benito et al., 2004; Brázdil et al., 2006) ou dados concretos (e.g. Seibert \& McDonnell, 2002, 2015; Arnold et al., 2015). Os dados provenientes de um linígrafo podem ser denominados como sistemáticos ou concretos, pois são dados quantitativos, mensurados continuamente ao longo do tempo com intervalo temporal pré-definido ( $\Delta \mathrm{t}=$ constante) em um determinado local (vetor posição $\vec{x}(\mathrm{x}, \mathrm{y}, \mathrm{z})$ ).

Os dados não sistemáticos (e.g. Francés et al., 1994; Benito et al., 2004; Brázdil et al., 2006) ou dados interpretativos (Seibert \& McDonnell, 2002, 2015; Arnold et al., 2015) são provenientes de uma grande variedade de outras fontes de dados que se caracterizam principalmente por se tratarem de registros sem regularidade. A representação matemática desse dado seria vetor posição $(\vec{x})$ e intervalo temporal $(\triangle \mathrm{t})$ diferente de constante. Esses dados podem ser exemplificados como relatos de moradores sobre áreas afetadas por determinada inundação, marca de cheia em uma construção e leitura pontual do nível do rio, respectivamente, exemplos de dado qualitativo, inferência quantitativa e dado quantitativo.

Para demonstrar as similaridades e unificar as terminologias, na Tabela 1 são apresentadas as características associadas às terminologias. No presente estudo, optou-se por usar a denominação dados sistemáticos e não sistemáticos.

\section{FONTES DE DADOS HIDROLÓGICOS}

Na hidrologia moderna, a fonte mais usual de dados para estudos hidrológicos se refere às estações de monitoramento que fornecem séries históricas dos dados mensurados (dados sistemáticos). No entanto, eventos hidrológicos extremos tanto de máxima quanto de mínima apresentam características atípicas aos demais elementos da série histórica acarretando em 
incremento nas incertezas dos dados (McMillan et al., 2018). Além disso, as condições do evento podem danificar parcial ou totalmente os equipamentos de medição ou ainda representar periculosidade ao operador do equipamento gerando falhas no registro. Assim, somente o uso de dados provenientes de estações de monitoramento dificulta a análise de processos hidrológicos ocorridos em eventos extremos. Nesse sentido, a combinação das diferentes fontes de dados mostra grande potencial na redução das incertezas e fornece uma visão holística sobre o evento passado (Brázdil et al., 2006).

Tabela 1 - Características dos dados sistemáticos e dados não sistemáticos.

\begin{tabular}{|c|c|}
\hline Dados sistemáticos & Dados não sistemáticos \\
\hline $\begin{array}{l}\text { - Intervalo temporal de registro pré-definido } \\
\text { resultando em série histórica }\end{array}$ & $\begin{array}{l}\text { - Intervalo temporal de registro irregular e } \\
\text { descontínuo }\end{array}$ \\
\hline - Local de registro constante ao longo do tempo & $\begin{array}{l}\text { - Local de registro não é constante ao longo do } \\
\text { tempo }\end{array}$ \\
\hline \multirow{3}{*}{ - Dados quantitativos registrados continuamente } & - Dados qualitativos \\
\hline & $\begin{array}{l}\text { - Inferências quantitativas a partir dos dados } \\
\text { qualitativos }\end{array}$ \\
\hline & - Dados quantitativos* \\
\hline - Posição: $\vec{x}=(\mathrm{x}, \mathrm{y}, \mathrm{z})$ & - Posição: $\vec{x} \neq$ constante \\
\hline - Intervalo temporal: $\Delta \mathrm{t}=$ constante & - Intervalo temporal: $\Delta \mathrm{t} \neq$ constante \\
\hline
\end{tabular}

*Registros pontuais realizados com o auxílio, direto ou indireto, de equipamento resultando em uma representação numérica (dado quantitativo), entretanto por não estar inserido em uma série histórica ocorrem incrementos na incerteza.

Ouarda et al. (1998) indicaram como tipos de fontes, além das estações de monitoramento: (a) evidência física dos eventos que deixaram vestígios na bacia hidrográfica, como perturbações do solo ou depósitos de sedimentos (paleo-hidrologia); (b) evidências botânicas dos eventos nas árvores, como anéis de crescimento, árvores inclinadas e outras anormalidades de crescimento (dendrohidrologia); e (c) observações registradas em jornais, arquivos etc. ou obtidos por meio de pesquisas com residentes da região. Já alguns autores empregam terminologias que remetem a escala temporal da inundação, ao invés de se referir a técnica de registro dos dados ou ao tipo de fonte.

Baker (1987), por exemplo, emprega a terminologia (a) pré-histórico, (b) histórico e (c) moderno, Segundo o autor, pré-históricos se referem aos eventos que ocorreram sem observação humana ou sem medição por procedimentos hidrológicos; eventos históricos são documentados por pessoas não profissionais da hidrologia; eventos modernos são aqueles com monitoramento contínuo por equipamentos destinados à hidrologia. Naulet (2002) também adotou uma classificação cronológica baseada em Baker (1987) e ilustrou as diferentes fontes de dados com o apoio de uma linha temporal. Brázdil et al. (2006) sugerem: (a) paleoinundação, (b) inundação histórica e (c) inundação instrumental.

Portanto, identifica-se que apesar das denominações empregadas remeterem a escala temporal do evento, os termos dos tipos de fontes se referem à forma como foi realizado o registro. Assim, uma denominação mais abrangente poderia esclarecer que essas diferentes fontes de dados hidrológicos não se limitam ao tempo em que o evento ocorreu, sendo também aplicadas para eventos recentes e, inclusive, utilizadas conjuntamente. A partir das denominações citadas, sugere-se o uso dos seguintes termos quanto à origem dos tipos de fonte:

a) Evidência instrumental: mensurações de variáveis, direta ou indiretamente com equipamentos e procedimentos técnicos associados à hidrologia;

b) Evidência natural: aspectos geomorfológicos, geológicos, sedimentológicos, botânicos, geoquímicos, dentre outros aspectos que indicam alterações no ambiente natural em decorrência do evento;

c) Evidência documental: registros inseridos em um contexto histórico e social, tais como documentos oficiais, notícias veiculadas pelos meios de comunicação, registros e/ou observações realizadas pela população.

Dentro deste contexto, a linha temporal para ilustrar as fontes de dados proposta por Naulet (2002) não é condizente. Assim, sugere-se a apresentação das fontes e classificação dos dados em um formato circular, como ilustrado na Figura 1. As fontes dos dados sistemáticos são evidências 
instrumentais provenientes de estações de monitoramento, sensores terrestres e sensores orbitais. Enquanto os dados não sistemáticos são provenientes das três fontes de dados: evidências naturais, documentais e instrumentais. Ressalta-se que as evidências instrumentais podem ser fontes de dados sistemáticos e não sistemáticos, dependendo da posição e do intervalo temporal. Por exemplo, quando consideramos o uso pontual de certo equipamento como o ADCP (Acoustic Doppler Current Profiler) para medir a vazão do rio durante certo evento obtém-se um dado não sistemático.

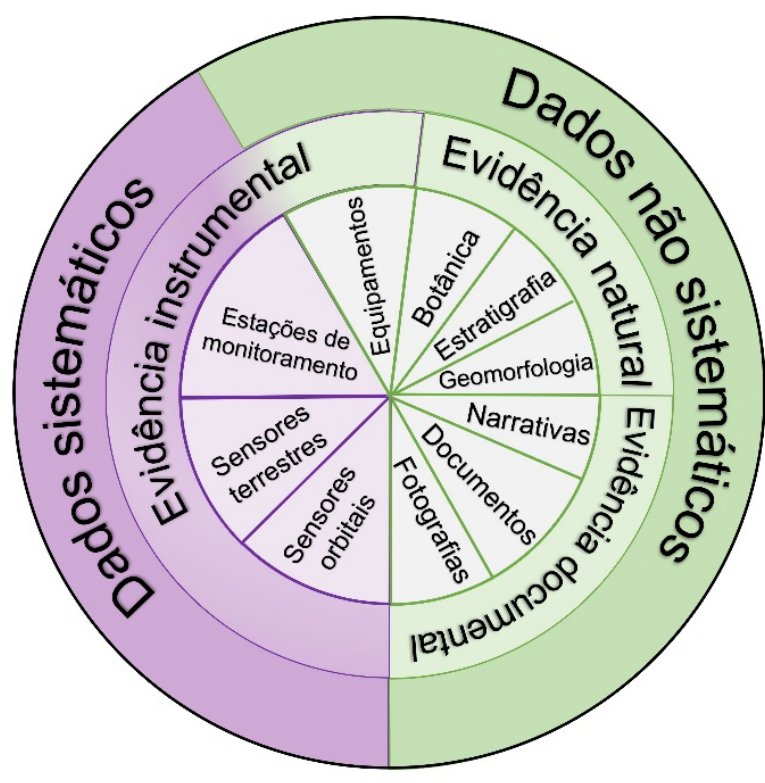

Figura 1 -Dados sistemáticos e não sistemáticos: exemplos de fontes de dados.

Nos próximos itens são descritas com maior detalhamento as diferentes fontes de dados.

\section{Evidência instrumental in-situ}

A evidência instrumental in-situ pode fornecer dados sistemáticos ou dados não sistemáticos dependendo das características descritas na Tabela 1. Apesar disso, em geral, essa fonte de dados é relacionada aos dados sistemáticos provenientes das estações de monitoramento. No Brasil, a ANA é responsável pelo Sistema Nacional de Informações sobre Recursos Hídricos (SNIRH) e pela coordenação da Rede Hidrometeorológica Nacional. A rede de monitoramento consiste em um conjunto de estações pluviométricas, fluviométricas, sedimentométricas, meteorológicas e de qualidade da água distribuídas sobre uma determinada região com o objetivo de caracterizar a bacia hidrográfica (Agência Nacional de Águas, 2014). Usualmente, as estações de monitoramento são compostas por estações pluviométricas e fluviométricas.

As estações pluviométricas fazem a mensuração da altura de chuva de forma automática por meio de pluviógrafos com intervalo de registro pré-definido (ex.: registro a cada $15 \mathrm{~min}$ ) ou pela leitura do valor acumulado em 24 horas por um operador em horário pré-fixado (ex.: às 7 horas nas estações da ANA). Já as estações fluviométricas medem níveis de água (também denominada como cota) e vazões. A mensuração do nível de água pode ser de forma automática continuamente ao longo do tempo ou por meio da leitura da régua linimétrica (Figura 2a) por um operador duas vezes ao dia (às 7 horas e às 17 horas nas estações da ANA) (Agência Nacional de Águas, 2014). Como a mensuração diária das vazões por um processo direto, como uso da ADCP (Figura 2b), seria excessivamente onerosa, difícil e perigosa, principalmente, durante eventos de cheia (Corato et al., 2014), o valor diário da vazão provém da estimativa de uma relação denominada curva-chave. Essa relação empírica utiliza um determinado intervalo de dados medidos em campo de nível de água (cota) e de vazão (descarga líquida), onde os valores inferiores ou superiores a faixa de dados medidos em campo são extrapolados. Apesar desse método ser bastante utilizado, muitos estudos apontam incertezas, principalmente na zona de extrapolação (e.g. Westerberg et al., 2010; Domeneghetti et al., 2012).

Apesar da participação do operador na mensuração de algumas variáveis, esses dados são sistemáticos e não se caracterizam como observação humana. Isso se justifica pelo registro ser feito 
com periodicidade determinada e com o uso de equipamentos destinados à medições hidrológicas. Os registros das estações automáticas, como pluviógrafos e linígrafos, são coletados por meio da conexão entre o datalogger e um computador portátil ou são transmitidos remotamente por sistema de telemetria.

Outros exemplos de fontes de evidências instrumentais se referem às medições de qualidade da água e de descarga sólida nos rios. Existem medições sistemáticas de parâmetros de qualidade da água, como Demanda Bioquímica de Oxigênio (DBO), Oxigênio Dissolvido (OD) e Temperatura, e medição de descarga sólida de sedimentos em suspensão. Medições de descarga sólida do leito, em geral, não são realizadas pela ANA no Brasil, embora este dado seja valioso para estimativas de assoreamento de reservatórios no contexto do país.

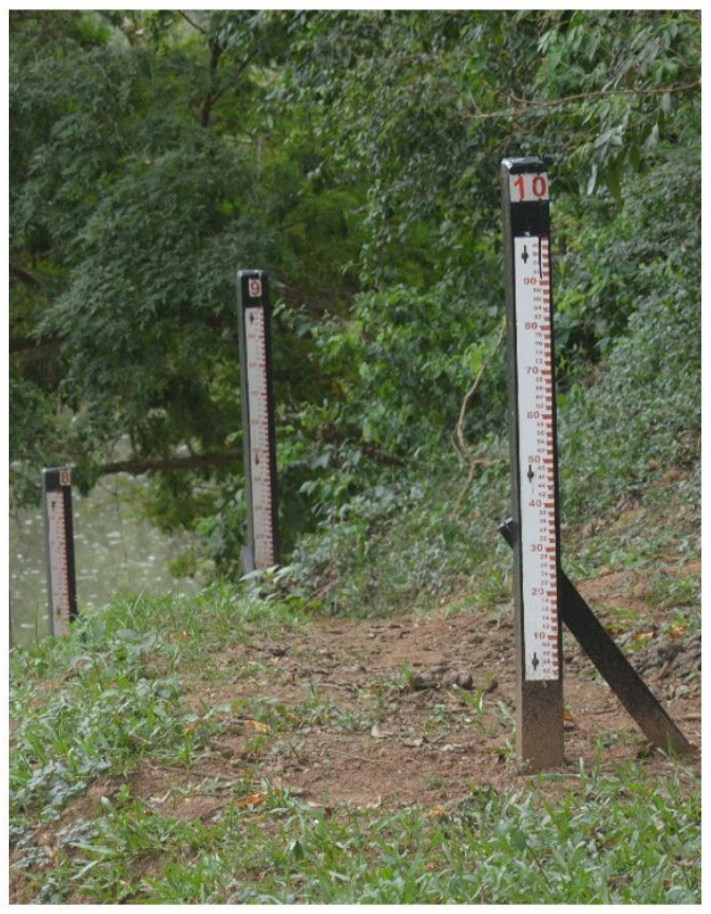

(a)

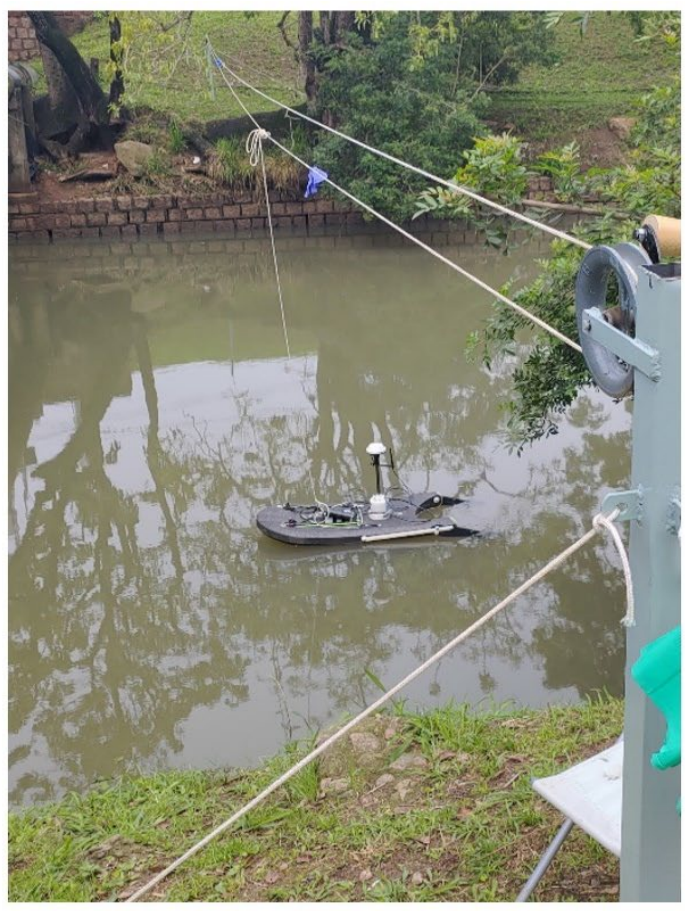

(b)

Figura 2 - Exemplo de evidência instrumental in-situ para determinação de vazão: (a) réguas linimétricas para estimativa da vazão pela curva-chave; (b) medidor de vazão ADCP (Acoustic Doppler Current Profiler).

Neste contexto, na Tabela 2 são elencadas algumas fontes de dados sistemáticos de variáveis hidrológicas e meteorológicas com abrangência nacional. Salienta-se que a lista não é exaustiva, contendo apenas alguns exemplos de base de dados de evidências instrumentais in-situ com abrangência em todo Brasil.

Tabela 2 - Evidência instrumental in-situ: fonte de dados sistemáticos com abrangência em todo Brasil

\begin{tabular}{c|c|c|c|c|c}
\hline Base de dados & Intervalo de & \multicolumn{3}{c}{ Dados } \\
\cline { 3 - 5 } Hidroweb (2020) & registro & P & H & Q \\
\hline Siário & $\mathrm{X}$ & $\mathrm{X}$ & $\mathrm{X}$ & \\
\hline Sistema HIDRO - Telemetria (Agência Nacional de Águas, 2020) & $15 \mathrm{~min}$ & $\mathrm{X}$ & $\mathrm{X}$ & $\mathrm{X}$ & \\
\hline $\begin{array}{c}\text { CEMADEN - Pluviômetros Automáticos (Centro Nacional de } \\
\text { Monitoramento e Alertas de Desastres Naturais, 2020a) }\end{array}$ & $10 \mathrm{a} 15 \mathrm{~min}$ & $\mathrm{X}$ & & & \\
\hline $\begin{array}{c}\text { CEMADEN - Estações Hidrológicas (Centro Nacional de } \\
\text { Monitoramento e Alertas de Desastres Naturais, 2020b) }\end{array}$ & $1 \mathrm{~h}$ & $\mathrm{X}$ & & & \\
\hline $\begin{array}{c}\text { Banco de Dados Meteorológicos para Ensino e Pesquisa BDMEP - } \\
\text { INMET (Instituto Nacional de Meterologia, 2020) }\end{array}$ & diário & $\mathrm{X}$ & & & $\mathrm{X}$ \\
\hline
\end{tabular}

$\mathrm{P}=$ Precipitação; $\mathrm{H}=$ Nível da água; $\mathrm{Q}=$ vazão líquida; $\mathrm{M}=$ variáveis meteorológicas, como temperatura do bulbo seco, temperatura do bulbo úmido, temperatura máxima, temperatura mínima, umidade relativa do ar, pressão atmosférica ao nível da estação, insolação, direção e velocidade do vento. 
Cabe ainda ressaltar que, no Brasil, em concordância com as legislações, os órgãos ambientais em todos os níveis - Federal, Estadual, e Municipal - exigem o monitoramento de variáveis ambientais em procedimentos de licenciamento ambiental e em condicionantes de licenças ambientais. Contudo, estes dados, geralmente, ficam restritos em relatórios entregues aos órgãos ambientais, para fazer o acompanhamento do empreendimento. Portanto, muitos dados não são disponibilizados ao amplo público de técnicos que poderiam utilizá-los em estudos e pesquisas na área de recursos hídricos.

\section{Evidência instrumental por sensores orbitais}

Os avanços tecnológicos incrementaram as fontes de dados, de modo que os dados hidrológicos também podem ser provenientes de produtos de sensoriamento remoto. Sensores embarcados em satélites fornecem imagens das quais é possível extrair dados hidrológicos. Exemplos de estudos que utilizaram ou que revisam o tipo de abordagem usando imagens são: Townsend (2001), Schumann et al. (2009), Zhang et al. (2014), Schumann \& Moller (2015), e Schumann \& Domeneghetti (2016).

Imagens de sensoriamento remoto podem ser obtidas de sensores passivos e ativos. Sensores passivos detectam a radiação natural emitida ou refletida pela superfície ou cena observada, sendo a luz solar a fonte mais comum de radiação medida. A maioria dos sistemas passivos utilizados em aplicações de sensoriamento remoto operam nas regiões do visível, infravermelho e micro-ondas. Sensores ativos, por outro lado, emitem radiação na direção do objeto a ser observado e detectam e medem a radiação refletida ou retroespalhada pelo alvo. A maioria dos sensores ativos opera na faixa de micro-ondas do espectro eletromagnético, o que os torna capazes de penetrar nas nuvens na maioria das condições (NASA EarthData, 2020). Os sensores ativos incluem instrumentos como o radar e o LIDAR (Light Detection and Ranging), sendo o Sentinel-1 e ALOS-PALSAR exemplos de satélites com sensores do tipo Radar de Abertura Sintética (SAR - Synthetic-aperture radar).

O uso de satélites geoestacionários para monitoramento de precipitações em conjunto com dados in-situ é reconhecido desde a década de 1970 (Rast et al., 2014). Na última década, o sensoriamento remoto por radar surgiu como uma ferramenta útil para apoiar e avançar a modelagem de inundações (Annis et al., 2020; Lee et al., 2020). O SAR foi considerado útil para mapas de inundação, pois os sinais de retorno do radar são normalmente baixos para corpos de águas sem vegetação, devido à reflexão da superfície da água. Essa característica permite que os limites de inundação sejam determinados com um bom nível de precisão em muitas condições (Teng et al., 2017). Notti et al. (2018) afirmam que a principal vantagem dos sensores SAR é a capacidade de obter imagens noturnas e sob diferentes condições climáticas, preenchendo assim a lacuna resultante de conjuntos de dados ópticos, que são corrompidos pela nebulosidade. Outra vantagem do SAR é a maior possibilidade de identificação de vegetação inundada (Hess et al., 1990). Entretanto, o SAR apresenta algumas desvantagens como o aumento do retroespalhamento do sinal devido a turbulência, vento e vegetação, dificultando ou impossibilitando a determinação da extensão da inundação em alguns casos (Teng et al., 2017).

Nessa linha, imagens de satélite, tanto ópticos quanto de radar, são fontes de dados sobre inundações. Novos lançamentos de satélite, como ALOS, RADARSAT-2, Terra-SAR-X, COSMO-SkyMed e Sentinel-1, que apresentam melhores sensores, resolução temporal mais curta, aquisição e processamento de imagens mais rápidos, resultaram em maior qualidade dos dados detectados. Esse aprimoramento dos dados ocorre desde o início dos anos 2000, tanto em questão de resolução quanto de disponibilidade (Teng et al., 2017; Notti et al., 2018).

A partir de sensores orbitais é possível obter uma gama de dados de monitoramento do ciclo hidrológico, incluindo: quantidade de água em uma região por gravimetria, umidade do solo, níveis de água em rios e reservatórios, áreas inundadas, evapotranspiração e precipitação. No caso dos dados de precipitação, é possível obter dados quantitativos registrados ao longo do tempo com periodicidade definida constituindo uma série histórica representativa de determinado local.

No entanto, essa fonte de dados também apresenta limitações na cobertura espacial e temporal. Além disso, ressalta-se que muitos destes dados são obtidos a partir de um processamento das imagens de satélites, e por este motivo eles possuem uma maior incerteza quando comparada aos dados medidos in-situ. A Tabela 3 apresenta uma lista não exaustiva com alguns exemplos de fontes de evidência instrumental por sensores orbitais. 
Tabela 3 - Exemplos de fontes de evidência instrumental por sensores orbitais: fonte de dados sistemáticos.

\begin{tabular}{|c|c|c|c|c|c|c|c|}
\hline \multirow{2}{*}{ Satélite ou Missão } & \multicolumn{7}{|c|}{ Dados } \\
\hline & $\mathbf{P}$ & $\mathbf{H}$ & $\mathbf{A}$ & I & $\mathbf{E}$ & $\mathbf{R}$ & $\mathbf{U}$ \\
\hline GRACE & & & $\mathrm{X}$ & & & & \\
\hline IceSat1 & & $\mathrm{X}$ & & & & & \\
\hline IceSat2 & & $\mathrm{X}$ & & & & & \\
\hline SWOT & & $\mathrm{X}$ & & & & & \\
\hline Jason & & $\mathrm{X}$ & & & & & \\
\hline GPM & $\mathrm{X}$ & & & & & & \\
\hline LandSat & & & & $\mathrm{X}$ & & $\mathrm{X}$ & \\
\hline Sentinel & & & & $\mathrm{X}$ & & $\mathrm{X}$ & \\
\hline Cbers & & & & $\mathrm{X}$ & & & \\
\hline SMOS & & & & & & & $\mathrm{X}$ \\
\hline MODIS & & & $\mathrm{X}$ & & $\mathrm{X}$ & $\mathrm{X}$ & \\
\hline
\end{tabular}

P= Precipitação; H= Nível da água; A= Quantidade de água por gravimetria; I= Áreas inundadas; E= Evapotranspiração; R= Reflectância da água; U= Umidade do solo.

\section{Evidência natural}

Enquanto as estações de monitoramento se limitam a curtos períodos de amostragem, as evidências naturais enfatizam os eventos ocorridos no passado identificando o padrão de recorrência (Stevaux, 2003). Os dados obtidos por esse tipo de fonte se referem a registros naturais, cuja participação do ser humano ocorre somente na análise e na interpretação dos dados (Baker, 2008). Esses dados são obtidos por sinais deixados pelo fluxo de água no ambiente e que são identificados por técnicas de botânica, sedimentologia, estratigrafia, dentre outras (Francés et al., 1994). Assim, desenvolve-se a paleo-hidrologia como uma área interdisciplinar com grande participação de pesquisadores da geomorfologia, pois esses dados são alterações na paisagem, nos sedimentos ou na vegetação que persistem após o evento hidrológico (Baker, 2008). Apesar desses dados serem associados, em geral, a paleoinundações, eles podem se referir a inundações recentes, seculares ou, até mesmo milenares (Baker, 1987).

Conforme Ouarda et al. (1998), o dado desse tipo de fonte é geralmente difícil de obter e, muitas vezes, é bastante impreciso. As três grandes incertezas apontadas por Benito et al. (2004) relacionadas às análises de vazões obtidas por essa fonte se referem à definição da profundidade da água atingida durante a inundação, a datação dos depósitos e a continuidade e completude dos registros. Conforme Guiming et al. (2016), existe dificuldade na preservação completa dos depósitos decorrentes das inundações em bacias hidrográficas com interferência humana. Portanto, o uso dessa técnica em bacias urbanizadas não é recomendado, sendo útil para áreas remotas (Baker, 1987). Além disso, essa fonte de dados requer o emprego de técnicas e equipamentos específicos o que pode representar altos custos e dificuldades na sua execução.

Fett Júnior (2011), por exemplo, estudou a evolução da paisagem no médio curso do Rio Pardo, localizado na região centro-leste do Rio Grande do Sul (RS) e forneceu importantes dados sobre o regime de cheias. A Figura 3 ilustra uma das seções estratigráficas analisadas pelo autor, localizada na margem direita do Rio Pardo, cerca de $8 \mathrm{~km}$ ao norte de Candelária, RS. A sucessão dos depósitos ilustra a movimentação lateral do rio quanto ao avanço e recuo das cheias, pois as variações no tamanho do grão podem ser atribuídas à distância em relação aos canais e a flutuações na energia de fluxo das cheias (Fett Júnior, 2011). 


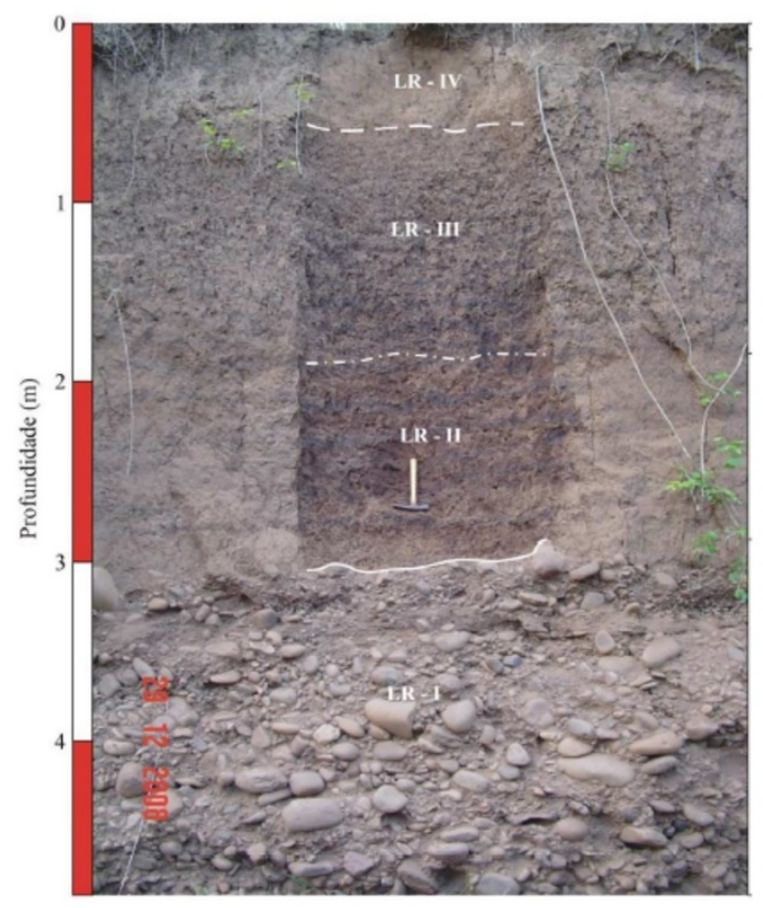

Figura 3 - Exemplo de evidência natural: estratigrafia em seção do médio curso do Rio Pardo/RS. Fonte: Fett Júnior (2011).

\section{Evidência documental}

Essa fonte de dados não sistemáticos se refere a registros, tais como observações, comunicações orais, documentos, registros audiovisuais, dentre outros, realizados pela população afetada pelo evento dos quais podem ser extraídos dados diretos ou indiretos (Brázdil et al., 2006). Exemplos de evidências documentais são registros em cartórios de óbitos, decretos de Situação de Emergência e/ou Estado de Calamidade Pública, notícias veiculadas nos meios de comunicação (Figura 4), marcas de cheia, livros, fotografias, narrativas, dentre outros. Assim, por vezes, para recuperar e analisar esse tipo de fonte de dados é necessário a cooperação dos profissionais da história. 0 termo hidrologia histórica é bastante empregado em estudos da União Europeia em que hidrólogos com a colaboração de historiadores buscam dados em registros históricos e elaboram base de dados para os estudos de reconstrução (Naulet et al., 2001), ilustrando a interdisciplinaridade desse campo de pesquisa.

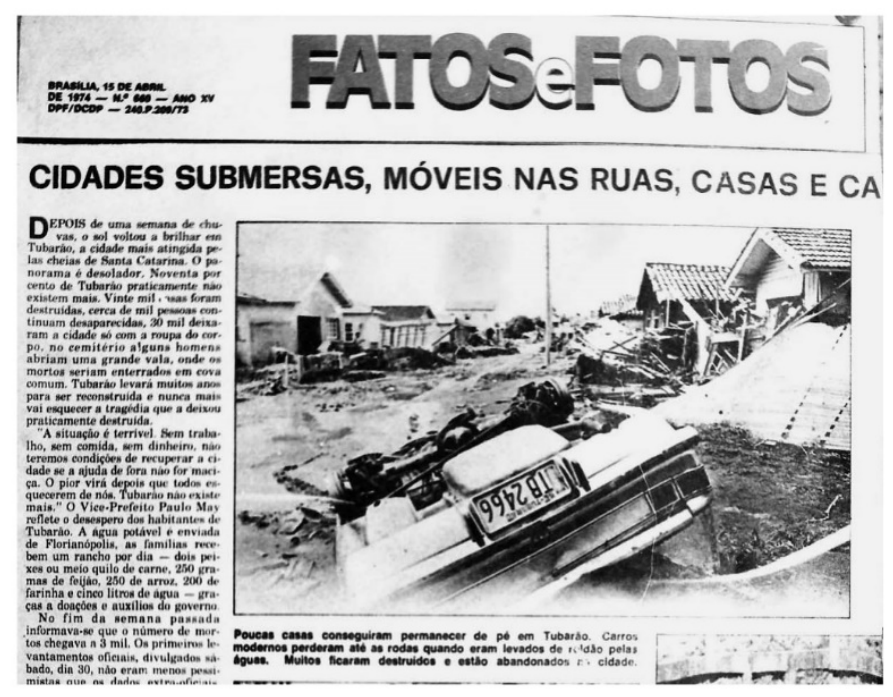

Figura 4 -Notícia veiculada em meio de comunicação de Brasília em 15/04/1974 relatando o evento ocorrido em março de 1974 no município de Tubarão/SC. Fonte: Patrimônio Histórico Municipal de Tubarão/SC. 
Apesar da utilidade dos dados provenientes de evidências documentais para a hidrologia, essencialmente, esses registros se referem à população que vivenciou o evento hidrológico. Conforme mencionado por Brázdil et al. (2006), esse tipo de dado contém a percepção humana do evento dentro de um contexto histórico. Assim como, as notícias veiculadas em jornais e revistas estão inseridas em um campo político-ideológico, seu conteúdo não é imparcial (Assunção, 2018). Para Gourley et al. (2013) podem ocorrer exageros nos relatos e notícias. No entanto, os dados provenientes de evidências documentais, quando existentes, fornecem informações valiosas, tais como tempo, forma e magnitude dos picos de inundação, que podem auxiliar na verificação do modelo computacional e acrescentar confiabilidade na representação do evento (Starkey etal., 2017), desde que os dados sejam analisados criticamente (Barriendos et al., 2014).

É importante ressaltar que em virtude da periculosidade dos eventos hidrológicos extremos, por vezes, ocorrem interações negativas com a população resultando em desastres hidrológicos de grande magnitude com danos econômicos, sociais e ambientais significativos. Considerando que a presença do ser humano é inerente à ocorrência do desastre (Kobiyama et al., 2018) e as falhas e as incertezas no registro de eventos extremos pelas estações de monitoramento, as evidências documentais podem fornecer detalhes importantes para compreensão dos eventos hidrológicos extremos. No Brasil, quando os eventos hidrológicos causam prejuízos significativos ao funcionamento da comunidade, as prefeituras devem fornecer dados sobre o desastre à plataforma Sistema Integrado de Informações sobre Desastres (2020). A utilização do S2ID para a solicitação de reconhecimento federal de Situação de Emergência ou Estado de Calamidade Pública passou a ser obrigatória com a Portaria №. 25, de 24 de janeiro de 2013 (Brasil, 2013). Nesse sistema, deve ser preenchido a Declaração Municipal de Atuação Emergencial e o Relatório fotográfico. Assim, nessa base de dados é possível, entre outros dados, buscar documentos oficiais que descrevem áreas afetadas, fotografias, dentre outras informações.

Além disso, as tecnologias emergentes, como VANTs e câmeras dos celulares, facilitam a produção de registros dos eventos pela população, enquanto a popularização do acesso à internet propicia a divulgação. Assim, além da busca em acervos históricos, as evidências documentais também estão disponíveis em meio eletrônico, tais como mídias sociais (e.g. Kryvasheyeu et al., 2016; De Andrade et al., 2017). Um exemplo de aplicação desse tipo de fonte de dados se encontra em Collischonn \& Kobiyama (2019). Para compreender um fenômeno hidrológico denominado cabeça d'água, os autores realizaram o levantamento de registros no Brasil em plataformas de vídeo e em notícias veiculadas na internet. Apesar dos autores apontarem algumas limitações dos dados dispersos geograficamente e fragmentados, - eles conseguiram identificar informações relevantes e identificar preliminarmente características da bacia e das condições associadas ao fenômeno. Portanto, como exemplificado, os dados qualitativos provenientes dessas observações humanas, apoiadas pelo uso de tecnologia, podem fornecer informações relevantes e, até mesmo, nortear os estudos mais avançados posteriores.

A maior participação da população na realização de registros relacionados a eventos hidrológicos impulsionou o aumento no número de estudos baseados em ciência cidadã (citizen science). De acordo com Buytaert et al. (2014), a ciência cidadã é definida como a participação da população (também denominada como não cientistas, não profissionais, leigos ou amadores) na produção de um novo conhecimento científico. Outras terminologias, além de ciência cidadã, também são utilizadas: informações geográficas voluntárias (volunteered geographical information - VGI), monitoramento participativo (participatory monitoring), colaboração coletiva (crowdsourcing) e observatório cidadão (citizen observatory) dependendo do grau de envolvimento da população e das técnicas adotadas (Starkey et al., 2017).

0 emprego desse conceito tem crescido na literatura científica desde 2010, apesar da prática em si ser muito mais antiga (Kullenberg \& Kasperowski, 2016; Njue et al., 2019). Kullenberg \& Kasperowski (2016) explanam que a ciência cidadã se origina de duas linhas principais no início da década de 1990. Nas ciências naturais com o americano Bonney e sua equipe, as contribuições dos voluntários consistem em observações, classificações e coletas dos dados que seriam posteriormente aplicados pelos cientistas em suas pesquisas. Enquanto nas ciências sociais, o britânico Irwin desenvolveu esse conceito visando o engajamento dos voluntários na construção do conhecimento para responder às suas necessidades e estendendo à tomada de decisão. No entanto, essas duas linhas principais de entendimento da ciência cidadã não esgotam todas as formas desse conceito (Kullenberg \& Kasperowski, 2016). Independente da origem, esse conceito expressa um caráter colaborativo entre os cientistas e a população. 
Apesar da relevância das observações humanas já ser reconhecida em outras áreas, como na biologia (Cunha et al., 2017), essa prática se expandiu na hidrologia moderna nos anos recentes (Starkey et al., 2017). No Brasil já existe um Sistema de Informação sobre a Biodiversidade Brasileira que incentiva a parceria entre "amadores e cientistas" no registro de dados, principalmente, para o monitoramento da fauna (Sistema da Informação sobre a Biodiversidade Brasileira, 2020). Dessa forma, ocorre a ampliação da área monitorada e maior quantidade de dados registrados contribuindo para os estudos dos especialistas. Na hidrologia existem exemplos de repositório como o Hydroshare (2020) apresentado por Tarboton et al. (2014). No Brasil, o aplicativo Hidromapp foi concebido para ser utilizado na forma de atividade pedagógica, possibilita o mapeamento colaborativo de informações sobre eventos hidrológicos (Oliveira et al., 2018). Também existem repositórios para dados topográficos, como o OpenTopography (2020), porém apesar de ter cobertura em todo o globo, esse repositório tem maior número de contribuições dos Estados Unidos da América.

Rocha (2019) identificou que os cientistas brasileiros empregam a ciência cidadã enfatizando o registro de dados, a garantia da confiabilidade e a viabilidade do uso para publicação de estudos científicos. Dentro desse contexto, a ciência cidadã se torna uma ferramenta para padronização da aquisição do dado, viabilizada pelo uso de aplicativos e demais recursos digitais. Mesmo que os registros sejam no formato numérico, esses dados são não sistemáticos, pois existe subjetividade e margem para interpretação.

Existe um valor nos dados provenientes das evidências documentais o qual vai além do uso desse dado no meio científico. A prática dos registros das observações humanas contribui para a manutenção da memória sobre o evento ocorrido. Livros, como de Silveira et al. (2009), Vettoretti (1992, 2004) são exemplos de registros dos eventos hidrológicos com texto descritivo que contribuem para preservação desses dados ao longo do tempo. A proximidade entre a população, os profissionais da hidrologia e o ambiente natural, como o rio, contribui para aumentar a conscientização. A conscientização é o primeiro passo em direção à construção de uma cultura de prevenção aos desastres, pois desperta o interesse da população, que por sua vez leva à atenção que conduz à ação (United Nations International Strategy for Disaster Reduction, 2007). A manutenção da memória social sobre os desastres, tais como inundações, torna a população mais preparada para eventos futuros (Viglione et al., 2014). De acordo com Vanelli \& Kobiyama (2019), a parceria entre profissionais da hidrologia e a comunidade local, muito mais do que apoiar o registro de dados, pode fornecer à comunidade condições de identificar uma situação de perigo iminente. Assim, o engajamento e a participação social da população, prioritariamente, podem contribuir para a autoproteção do indivíduo devido ao aprimoramento de sua percepção do risco.

\section{USO INTEGRADO DE DADOS SISTEMÁTICOS E NÃO SISTEMÁTICOS NA HIDROLOGIA}

O principal ponto de discussão sobre o valor de uso dos dados não sistemáticos em comparação com os sistemáticos em Blöschl et al. (2019) se refere a maior variabilidade espacial intrínseca aos não sistemáticos, questionando em quais situações pode ocorrer substituição do tempo pelo espaço. De acordo com os autores, o potencial desses dados ainda não foi totalmente explorado pela comunidade científica da hidrologia.

Ainda hoje, dentro do contexto positivista da hidrologia moderna, assume-se que os dados sistemáticos apresentam maior confiabilidade do que os dados não sistemáticos. Isso pode ser justificada pela hidrologia moderna ser pautada em dados e métodos quantitativos. Assim, são creditadas maiores incertezas aos dados não sistemáticos, em especial aos qualitativos, de modo que, em geral, são requeridas inferências quantitativas para fazer uso desses dados.

Diferentemente da ideia de substituição, esse estudo propõe a consolidação do uso integrado de dados sistemáticos e não sistemáticos. A incorporação de dados não sistemáticos nos estudos hidrológicos não é recente. Contudo, conforme Blöschl et al. (2019) ainda não existe um consenso no uso desse tipo de dado. Autores como Baker (1987), Francés et al. (1994), Fernandes et al. (2013), Barriendos et al. (2014), dentre outros, demonstraram que a curta extensão temporal das séries históricas pode ser complementada com dados não sistemáticos contribuindo para análises de probabilidade de ocorrência.

O uso de dados sistemáticos e não sistemáticos de forma integrada já ocorre em alguns estudos hidrológicos. Estudos recentes (e.g.: Archer et al., 2019; Brázdil et al., 2019; Sy et al., 2020; e Vanelli et al., 2020) demonstraram a importância de associar o uso de dados sistemáticos e não sistemáticos para o estudo de eventos hidrológicos extremos passados. Desde 2007, a União Europeia (UE) reconhece a importância de extrair informações das inundações significativas já ocorridas para melhorar o planejamento (União 
Europeia, 2007). De acordo com Vanelli et al., (2020), no Brasil, ainda não existem normativas, como na UE, que incentivam a investigação de eventos passados para apoiar a gestão de risco e de desastres. Esse tipo de estudo, denominado reconstrução por Balasch et al. (2010), Barriendos et al. (2014) e Vanelli et al. (2020), entre outros, refere-se a representar um certo evento ocorrido visando determinar os fatores associados aos processos físicos e avaliar as interações entre as variáveis possibilitando uma melhor compreensão dos mecanismos envolvidos (Fortunato et al. 2017; Velásquez et al., 2020). Entretanto, outros termos já foram utilizados com o mesmo propósito: retromodelagem (Remo \& Pinter, 2007; Balasch et al., 2011) e reconstituição (Ribeiro Neto et al., 2015).

Existe um grande potencial de uso dos dados não sistemáticos para compreensão dos eventos hidrológicos extremos e também para demais estudos hidrológicos com baixa quantidade ou qualidade de dados sistemáticos, como para bacias não monitoradas, ou com baixa densidade de estações de monitoramento ou cuja discretização temporal não é adequada para representar a rápida resposta hidrológica. Além disso, apesar da socio-hidrologia ter sido apresentada por Sivapalan et al. (2012) como "uma nova ciência interdisciplinar mas quantitativa sobre pessoas e águas", pesquisadores como Mondino et al. (2020) e Mostert (2018) demonstram que o uso de dados não sistemáticos nos estudos sobre as interações bidirecionais do sistema ser humano-água é de grande relevância.

Neste contexto, questiona-se como aumentar a credibilidade dos dados não sistemáticos visando incorporá-los aos estudos das ciências das águas. Recursos associados à inteligência artificial têm auxiliado no processamento e uso de uma grande variedade de dados para aplicações na hidrologia moderna (e.g. Lucchese et al., 2020; Kadam et al., 2019; Firat, 2008). Entretanto, alguns dados relevantes por não se enquadrarem em um mesmo padrão podem ser excluídos da análise pelos algoritmos.

Assim, estimula-se a busca pelos métodos já consolidados nas ciências sociais e humanas para apoiar o uso dos dados não sistemáticos, desde a aquisição, como por exemplo em uma entrevista em campo, até a análise dos dados. Não se trata de valorizar os dados não sistemáticos em detrimento dos dados sistemáticos, e sim de renovar a hidrologia com uma abordagem que combine ambos os tipos de dados e métodos.

De acordo com Sampieri et al. (2013), a abordagem mista, ou seja, uso de dados e métodos qualitativos e quantitativos, surge como consequência da necessidade de enfrentar a complexidade dos problemas de investigação que ocorrem em todas as ciências e abordar de forma holística. Os autores afirmaram que o enfoque misto foi impulsionado no final da década de 1970 por dois estudiosos: Trend (1978) e Jick (1979). Ambos os trabalhos se referiram ao método de triangulação, anteriormente aplicado em métodos qualitativos, mas que os autores propuseram para coletar e analisar dados nas perspectivas dos métodos quantitativos e qualitativos. 0 método de triangulação possibilita examinar o mesmo fenômeno de múltiplas perspectivas, enriquecendo a compreensão do fenômeno, produzindo explicações mais sólidas e incrementando a validade das análises e das inferências (Trend, 1978; Jick, 1979; Minayo, 2012).

Os diferentes dados disponíveis podem ser analisados de forma crítica e conjunta tendo o evento ocorrido (realidade) como alvo (Figura 5). Quando as informações extraídas dos dados são dispersas e não convergem para representação do evento ocorrido, os dados podem ser considerados não confiáveis e não válidos (Figura 5a). Os dados podem gerar informações consistentes entre si, porém as informações não são úteis para representar o evento ocorrido (Figura 5b). Espera-se que quando analisados criticamente, os dados sistemáticos e não sistemáticos de diferentes fontes (evidências instrumental, natural e documental) sejam capazes de fornecer informações válidas e confiáveis, ou seja, consistentes entre si e que convirjam para a representação do evento (Figura 5c).
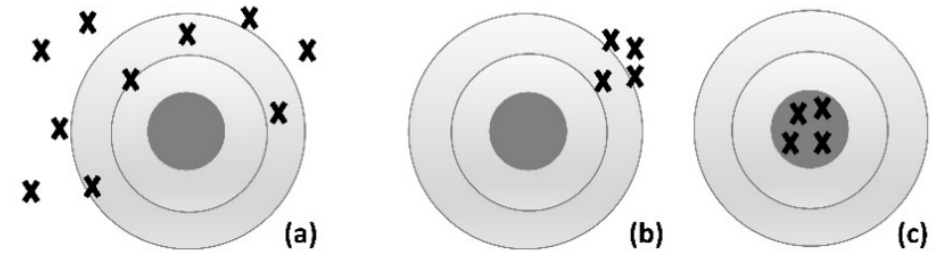

Figura 5-Representação da validade e confiabilidade dos dados em analogia com tiro ao alvo. 
Para exemplificar, pode ser citado o estudo de reconstrução da inundação catastrófica ocorrida em 1974 no Rio Tubarão, no estado de Santa Catarina, realizado por Vanelli et al. (2020). Os autores ao analisarem os dados de chuva e vazão das estações de monitoramento identificaram que os valores não eram condizentes com a proporção do evento ocorrido. A busca de outras fontes de dados em evidências documentais, tais como Machado (2005), Vettoretti $(1992,2004)$, foram essenciais para o estudo de reconstrução do evento. Os autores demonstraram que o uso de dados qualitativos, na ausência de dados quantitativos válidos e confiáveis, contribuiu para a modelagem, similarmente ao que afirmaram Seibert \& McDonnell (2015). Outros estudos de reconstrução, como de Ruiz-Bellet et al. (2015), Brázdil et al. (2019), Sy et al. (2020) também indicaram o potencial de extrair informações relevantes das evidências documentais.

\section{CONCLUSÕES E PROPOSTAS}

A proposição de Blöschl et al. (2019) sobre a utilidade de dados não sistemáticos em relação aos dados provenientes de estações de monitoramento como um dos 23 problemas não solucionados na hidrologia motivou a revisão sobre dados e diferentes tipos de fontes para estudos hidrológicos. Os dados são o ponto de partida das pesquisas e, consequentemente, para o avanço no conhecimento. 0 desenvolvimento da hidrologia moderna sob a perspectiva quantitativa se sobrepôs à aplicação de métodos qualitativos. Apesar de fontes alternativas às estações de monitoramento serem discutidas desde a década de 1970, principalmente, ao que se refere eventos hidrológicos extremos de máxima, o questionamento de Blöschl et al. (2019) denota que ainda não existe consenso na comunidade da hidrologia.

Neste contexto, o presente estudo, inicialmente, apresentou a classificação dos dados, visto que diferentes autores utilizam terminologias distintas e foi proposto o uso da denominação dados sistemáticos e dados não sistemáticos. Os dados sistemáticos são dados quantitativos, mensurados continuamente ao longo do tempo com intervalo temporal pré-definido $(\Delta t=$ constante $)$ em um determinado local (vetor posição $\vec{x}(\mathrm{x}, \mathrm{y}, \mathrm{z})$ ). Os dados provenientes de estações de monitoramento e de sensores orbitais são exemplos de dados sistemáticos. Enquanto os dados não sistemáticos podem ser provenientes de diferentes fontes e se caracterizam, principalmente, por registros sem regularidade, ou seja, $\vec{x}$ e $\triangle$ t variáveis.

As fontes de dados hidrológicos podem ser classificadas conforme a origem dos tipos de fonte: evidências instrumentais, evidências naturais e evidências documentais. Essas diferentes fontes fornecem dados distintos devido às características do registro. As evidências instrumentais são mensurações de variáveis realizadas direta ou indiretamente com equipamentos e procedimentos técnicos associados à hidrologia. Por se tratarem de mensurações fornecem dados quantitativos, entretanto podem ser dados sistemáticos ou não sistemáticos, dependendo se o registro for realizado em local e periodicidade definida ou não.

Já nas evidências naturais e documentais, o registro do dado não é padronizado, fornecendo dados interpretativos. As indicações do evento no ambiente natural devem ser interpretadas pelo uso de técnicas específicas para extração dos dados. Enquanto os registros realizados pelo ser humano, independente do formato da fonte: narrativas, documentos, fotografias, dentre outros, inserem-se em um contexto histórico e social. Essa pluralidade das metodologias empregadas para o registro e dos dados representa uma das grandes dificuldades no uso desses tipos de fonte na hidrologia sob a perspectiva atual.

Contudo, o uso integrado de diferentes fontes de dados, além de úteis para compreensão de eventos hidrológicos extremos, também são relevantes para bacias não monitoradas, ou com baixa densidade de estações de monitoramento ou cuja discretização temporal não é suficiente para representar a rápida resposta hidrológica, como por exemplo, em bacias montanhosas ou de pequena área de drenagem. Inclusive, a maior disponibilidade de acesso à internet e à tecnologia atual proporcionada pelos celulares representa um grande potencial no aumento de dados provenientes de evidências documentais.

O uso integrado de dados sistemáticos e não sistemáticos fornece uma visão mais ampla dos fenômenos. Assim, os conhecimentos já estabelecidos em outras ciências podem contribuir para o avanço do uso integrado de dados sistemáticos e não sistemáticos na hidrologia. Inclusive, pode ser considerado essencial para ampliar os conhecimentos sobre as interações bidirecionais entre a sociedade e água. Para isso, o método de triangulação é uma técnica que pode ser aplicada visando 
testar a validade e confiabilidade dos dados sistemáticos e não sistemáticos provenientes de diferentes fontes para a representação do fenômeno ocorrido (realidade).

Estudos de eventos hidrológicos extremos passados já fazem uso de dados não sistemáticos e demonstram a relevância do uso integrado dos dados. Assim, olhar para o passado e compreender as diferentes fontes de dados que podem ser aplicados nos estudos hidrológicos possibilita que a aquisição e o armazenamento dos dados sobre os eventos atuais sejam mais adequados ao seu uso futuro.

Além de fomentar o uso integrado de dados sistemáticos e não sistemáticos, o presente estudo finaliza apresentando propostas que podem aumentar a disponibilidade de dados hidrológicos para toda a comunidade de técnicos e pesquisadores no Brasil:

- Ampliar o uso de repositórios digitais colaborativos, cuja inserção dos dados seja realizada por indivíduos, sejam profissionais ou leigos. Cada indivíduo é capaz de enviar os seus registros, bem como visualizar os demais registros mediante a realização de um cadastro simplificado. Em um sistema autogerido, os usuários (população e pesquisadores) podem avaliar o dado conforme critérios pré-definidos e, até mesmo, reportar dados.

- Disponibilizar um banco de dados de acesso público a partir dos dados provenientes de monitoramento exigido pelas legislações ambientais, possibilitando o uso para concepção e/ou validação de projetos de engenharia, bem como apoiar pesquisas científicas.

- Permitir que os bancos de dados sistemáticos existentes possam incorporar dados de monitoramento sistemático realizados por outras companhias e usuários a serem cadastrados na rede, por exemplo, grupos de pesquisa de universidades. Estes dados poderiam ser identificados com codificação específica, indicando sua origem de terceiros, isentando a responsável pelo banco de dados de realizar a verificação da qualidade destes dados.

\section{AGRADECIMENTOS}

Os autores agradecem ao Conselho Nacional de Desenvolvimento Científico (CNPq) pelo apoio financeiro ao desenvolvimento da pesquisa (Processos números 141384/2019-0 e 305636/2019-7). Este estudo é parte de um projeto científico apoiado pela Coordenação de Aperfeiçoamento de Pessoal de Nível Superior (CAPES) [Código Financiamento 001].

\section{REFERÊNCIAS}

Agência Nacional de Águas - ANA. (2014). Medição de descarga líquida em grandes rios: manual técnico. (2. ed.). Brasília, DF: ANA.

Agência Nacional de Águas - ANA. (2020). Sistema HIDRO - Telemetria. Recuperado em 5 de outubro, de http://www.snirh.gov.br/hidrotelemetria/Mapa.aspx

Annis, A., Nardi, F., Volpi, E., \& Fiori, A. (2020). Quantifying the relative impact of hydrological and hydraulic modelling parameterizations on uncertainty of inundation maps. Hydrological Sciences Journal, 65(4), 507 523. http://dx.doi.org/10.1080/02626667.2019.1709640.

Archer, D., O'Donnell, G., Lamb, R., Warren, S., \& Fowler, H. J. (2019). Historical flash floods in England: new regional chronologies and database. Journal of Flood Risk Management, 12526, 1-14. http://dx.doi.org/10.1111/jfr3.12526.

Arnold, J. G., Youssef, M. A., Yen, H., White, M. J., Sheshukov, A. Y., Sadeghi, A. M., Moriasi, D. N., Steiner, J. L., Amatya, D. M., Skaggs, R. W., Haney, E. B., Jeong, J., Arabi, M., \& Gowda, P. H. (2015). Hydrological processes and model representation: impact of soft data on calibration. American Society of Agricultural and Biololgical Engineers, 58(6), 1637-1660.

Assunção, V. K. (2018). Memórias da enchente de 1974 e produção do espaço em Tubarão (SC). Mercator (Fortaleza), 17, 1-16. http://dx.doi.org/10.4215/rm2018.e17001.

Baker, V. R. (1987). Paleoflood hydrology and extraordinary flood events. Journal of Hydrology (Amsterdam), 96, 79-99.

Baker, V. R. (2008). Paleoflood hydrology: origin, progress, prospects. Geomorphology, 101, 1-13.

Balasch, J. C., Ruiz-Bellet, J. L., \& Tuset, J. (2011). Historical flash floods retromodelling in the Ondara River in Tàrrega (NE Iberian Peninsula). Natural Hazards and Earth System Sciences, 11, 3359-3371. http://dx.doi.org/10.5194/nhess-11-3359-2011. 
Balasch, J. C., Ruiz-Bellet, J. L., Tuset, J., \& Oliva, J. M. (2010). Reconstruction of the 1874 Santa Tecla's rainstorm in Western Catalonia (NE Spain) from flood marks and historical accounts. Natural Hazards and Earth System Sciences, 10, 2317-2325. http://dx.doi.org/10.5194/nhess-10-2317-2010.

Barriendos, M., Ruiz-Bellet, J. L., Tuset, J., Mazón, J., Balasch, J. C., Pino, D., \& Ayala, J. L. (2014). The "Prediflood” database of historical floods in Catalonia (NE Iberian Peninsula) AD 1035-2013, and its potential applications in flood analysis. Hydrology and Earth System Sciences, 18, 4807-4823. http://dx.doi.org/10.5194/hess-18-4807-2014.

Benito, G., Lang, M., Barriendos, M., Llasat, M. C., Francés, F., Ouarda, T., Thorndycraft, V. R., Enzel, Y., Bardossy, A., Coeur, D., \& Bobée, B. (2004). Use of systematic, palaeoflood and historical data for improvement of flood risk estimation. Review of scientific methods. Natural Hazards, 31, 623-643.

Blöschl, G., Bierkens, M. F. P., Chambel, A., Cudennec, C., Destouni, G., Fiori, A., Kirchner, J. W., McDonnell, J. J., Savenije, H. H. G., Sivapalan, M., Stumpp, C., Toth, E., Volpi, E., Carr, G., Lupton, C., Salinas, J., Széles, B., Viglione, A., Aksoy, H., Allen, S. T., Amin, A., Andréassian, V., Arheimer, B., Aryal, S. K., Baker, V., Bardsley, E., Barendrecht, M. H., Bartosova, A., Batelaan, O., Berghuijs, W. R., Beven, K., Blume, T., Bogaard, T., Borges de Amorim, P., Böttcher, M. E., Boulet, G., Breinl, K., Brilly, M., Brocca, L., Buytaert, W., Castellarin, A., Castelletti, A., Chen, X., Chen, Y., Chen, Y., Chifflard, P., Claps, P., Clark, M. P., Collins, A. L., Croke, B., Dathe, A., David, P. C., de Barros, F. P. J., de Rooij, G., Di Baldassarre, G., Driscoll, J. M., Duethmann, D., Dwivedi, R., Eris, E., Farmer, W. H., Feiccabrino, J., Ferguson, G., Ferrari, E., Ferraris, S., Fersch, B., Finger, D., Foglia, L., Fowler, K., Gartsman, B., Gascoin, S., Gaume, E., Gelfan, A., Geris, J., Gharari, S., Gleeson, T., Glendell, M., Gonzalez Bevacqua, A., González-Dugo, M. P., Grimaldi, S., Gupta, A. B., Guse, B., Han, D., Hannah, D., Harpold, A., Haun, S., Heal, K., Helfricht, K., Herrnegger, M., Hipsey, M., Hlaváčiková, H., Hohmann, C., Holko, L., Hopkinson, C., Hrachowitz, M., Illangasekare, T. H., Inam, A., Innocente, C., Istanbulluoglu, E., Jarihani, B., Kalantari, Z., Kalvans, A., Khanal, S., Khatami, S., Kiesel, J., Kirkby, M., Knoben, W., Kochanek, K., Kohnová, S., Kolechkina, A., Krause, S., Kreamer, D., Kreibich, H., Kunstmann, H., Lange, H., Liberato, M. L. R., Lindquist, E., Link, T., Liu, J., Loucks, D. P., Luce, C., Mahé, G., Makarieva, O., Malard, J., Mashtayeva, S., Maskey, S., Mas-Pla, J., MavrovaGuirguinova, M., Mazzoleni, M., Mernild, S., Misstear, B. D., Montanari, A., Müller-Thomy, H., Nabizadeh, A., Nardi, F., Neale, C., Nesterova, N., Nurtaev, B., Odongo, V. O., Panda, S., Pande, S., Pang, Z., Papacharalampous, G., Perrin, C., Pfister, L., Pimentel, R., Polo, M. J., Post, D., Prieto Sierra, C., Ramos, M.-H., Renner, M., Reynolds, J. E., Ridolfi, E., Rigon, R., Riva, M., Robertson, D. E., Rosso, R., Roy, T., Sá, J. H. M., Salvadori, G., Sandells, M., Schaefli, B., Schumann, A., Scolobig, A., Seibert, J., Servat, E., Shafiei, M., Sharma, A., Sidibe, M., Sidle, R. C., Skaugen, T., Smith, H., Spiessl, S. M., Stein, L., Steinsland, I., Strasser, U., Su, B., Szolgay, J., Tarboton, D., Tauro, F., Thirel, G., Tian, F., Tong, R., Tussupova, K., Tyralis, H., Uijlenhoet, R., van Beek, R., van der Ent, R. J., van der Ploeg, M., Van Loon, A. F., van Meerveld, I., van Nooijen, R., van Oel, P. R., Vidal, J.-P., von Freyberg, J., Vorogushyn, S., Wachniew, P., Wade, A. J., Ward, P., Westerberg, I. K., White, C., Wood, E. F., Woods, R., Xu, Z., Yilmaz, K. K., \& Zhang, Y. (2019). Twenty-three unsolved problems in hydrology - a community perspective. Hydrological Sciences Journal, 64(10), 1141-1158. http://dx.doi.org/10.1080/02626667.2019.1620507.

Brasil. (2013). Portaria no 25, de 24 de janeiro de 2013. Altera a Portaria no 526, de 6 de setembro de 2012, publicada no Diário Oficial da União no dia 10 de setembro de 2012, para incluir o marco inicial de obrigatoriedade de utilização do Sistema Integrado de Informações sobre Desastres - S2ID. Diário Oficial [da] República Federativa do Brasil, Brasília.

Brázdil, R., \& Kundzewicz, Z. W. (2006). Historical hydrology: editorial. Hydrological Sciences Journal, 51(5), 733738. http://dx.doi.org/10.1623/hysj.51.5.733.

Brázdil, R., Demarée, G. R., Kiss, A., Dobrovolný, P., Chromá, K., Trnka, M., Dolák, L., Reznícková, L., Zahradnícek, P., Limanowka, D., \& Jourdain, S. (2019). The extreme drought of 1842 in Europe as described by both documentary data and instrumental measurements. Climate of the Past, 15, 1861-1884. http://dx.doi.org/10.5194/cp-15-1861-2019.

Brázdil, R., Kundzewicz, Z. W., \& Benito, G. (2006). Historical hydrology for studying flood risk in Europe. Hydrological Sciences Journal, 51(5), 739-764. http://dx.doi.org/10.1623/hysj.51.5.739.

Brym, R. J., Lie, J., Hamlin, C. L., Mutzenberg, R., Soares, E. V., \& Maior, H. P. S. (2006). Sociologia: sua bússola para um novo mundo. São Paulo: Thomson Learning.

Buytaert, W., Zulkafli, Z. , Grainger, S., Acosta, L., Alemie, T. C., Bastiaensen, J., De Bièvre, B., Bhusal, J., Clark, J., Dewulf, A., Foggin, M., Hannah, D. M., Hergarten, C., Isaeva, A., Karpouzoglou, T., Pandeya, B., Paudel, D., Sharma, K., Steenhuis, T., Tilahun, S., Van Hecken, G., \& Zhumanova, M. (2014). Citizen science in hydrology and water resources: opportunities for knowledge generation, ecosystem service management, and sustainable development. Frontiers of Earth Science, 2, 1-21. http://dx.doi.org/10.3389/feart.2014.00026.

Centro Nacional de Monitoramento e Alertas de Desastres Naturais - CEMADEN. (2020a). Pluviômetros automáticos. Recuperado em 5 de outubro, de http://www.cemaden.gov.br/mapainterativo/ 
Centro Nacional de Monitoramento e Alertas de Desastres Naturais - CEMADEN. (2020b). Estações hidrológicas. Recuperado em 5 de outubro, de http://www.cemaden.gov.br/mapainterativo/

Collischonn, W., \& Kobiyama, M. (2019). A hidrologia da cabeça d’água (1): ocorrências e observações no Brasil. In: Anais do $23^{\circ}$ Simpósio Brasileiro de Recursos Hídricos (SBRH). Foz do Iguaçu, PR.

Corato, G., Ammari, A., \& Moramarco, T. (2014). Conventional point-velocity records and surface velocity observations for estimating high flow discharge. Entropy (Basel, Switzerland), 16(10), 5546-5559.

Cunha, D. G. F., Marques, J. F., Resende, J. C., Falco, P. B., Souza, C. M., \& Loiselle, S. A. (2017). Citizen science participation in research in the environmental sciences: key factors related to projects' success and longevity. Anais da Academia Brasileira de Ciências, 89, 2229-2245. http://dx.doi.org/10.1590/00013765201720160548.

De Andrade, S. C., Restrepo-Estrada, C., Delbem, A. C. B., Mendiondo, E. M., \& De Albuquerque, J. P. (2017). Mining rainfall spatio-temporal patterns in twitter: a temporal approach. Lecture notes. Geoinformation and cartography (pp. 19-37). Springer International Publishing. https://doi.org/10.1007/978-3-319-567594_2.

Domeneghetti, A., Castellarin, A., \& Brath, A. (2012). Assessing rating-curve uncertainty and its effects on hydraulic model calibration. Hydrology and Earth System Sciences, 16, 1191-1202.

Dooge, J. C. I. (2004). Background to modern hydrology. The Basis of Civilization - Water Science?. In: Proceedings of the UNESCO/IAHS/IWHA Symposium. Rome.

Fernandes, W., Naghettini, M., \& Loschi, R. (2013). Método para a estimação de quantis de enchentes extremas com o emprego conjunto de análise bayesiana, de informações não sistemáticas e de distribuições limitadas superiormente - Parte 2: aplicação. Revista Brasileira de Recursos Hídricos, 18(2), 189-199.

Fett Júnior, N. (2011). Aloestratigrafia e evolução do relevo do Pleistoceno médio ao Holoceno no médio curso do Rio Pardo, região centro-leste do Estado do Rio Grande do Sul, Brasil (Tese de doutorado). Universidade Federal de Santa Catarina, Florianópolis.

Firat, M. (2008). Comparison of Artificial Intelligence Techniques for river flow forecasting. Hydrology and Earth System Sciences, 12, 123-139.

Fortunato, A., Freire, P., Bertin, X., Rodrigues, M., Ferreira, J., \& Liberato, M. L. R. (2017). A numerical study of the February 15, 1941 storm in the Tagus estuary. Continental Shelf Research, 144, 50-64.

Francés, F., Salas, J. D., \& Boes, D. C. (1994). Flood frequency analysis with systematic, historical and paleoflood data based on the general extreme value models. Water Resources Research, 30, 1653-1664.

Gourley, J. J., Hong, Y., Flamig, Z. L., Arthur, A., Clark, R., Calianno, M., Ruin, I., Ortel, T., Wieczorek, M. E., Kirstetter, P.-E., Clark, E., \& Krajewski, W. F. (2013). A unified flash flood database across the United States. Bulletin of the American Meteorological Society, 94(6), 799-805. http://dx.doi.org/10.1175/BAMS-D-1200198.1.

Guiming, H., Chun, C. H., Yali, Z., Jiangli, P., Xiaochun, Z., Yongqiang, G., Yuzhu, Z., \& Xueru, Z. (2016). Hydrological studies of the historical and palaeoflood events on the middle Yihe River, China. Geomorphology, 274, 152161. http://dx.doi.org/10.1016/j.geomorph.2016.09.004

Herzog, D. (2015). Data Literacy: a user's guide (224 p.). USA: Sage Publications/Missouri School of Journalism.

Hess, L. L., Melack, J. M., \& Simonett, D. S. (1990). Radar detection of flooding beneath the forest canopy: a review. International Journal of Remote Sensing, 11(7), 1313-1325. http://dx.doi.org/10.1080/01431169008955095.

Hidroweb. (2020). Recuperado em 5 de outubro, de http://www.snirh.gov.br/hidroweb/apresentacao

Horton, R. E. (1931). The field, scope, and status of the science of hydrology. Eos (Washington, D.C.), 12(1), 189202. http://dx.doi.org/10.1029/TR012i001p00189-2.

Hydroshare. (2020). Recuperado em 5 de outubro, de https://www.hydroshare.org/

Instituto Nacional de Meterologia - INMET. (2020). Banco de Dados Meteorológicos para Ensino e Pesquisa BDMEP. Recuperado em 5 de outubro, de https://bdmep.inmet.gov.br/

Jick, T. D. (1979). Mixing qualitative and quantitative methods: triangulation in Action. Administrative Science Quarterly, 24(4), 602-611.

Kadam, A., Karnewar, A. S., Umrikar, B., \& Sankhua, R. N. (2019). Hydrological response-based watershed prioritization in semiarid, basaltic region of western India using frequency ratio, fuzzy logic and AHP method. Environment, Development and Sustainability, 21, 1809-1833. http://dx.doi.org/10.1007/s10668018-0104-4. 
Kaiser, M., Günnemann, S., \& Disse, M. (2020). Providing guidance on efficient flash flood documentation: an application based approach. Journal of Hydrology (Amsterdam), 581, 1-15. http://dx.doi.org/10.1016/j.jhydrol.2019.124466.

Kidd, C., Becker, A., Huffman, G. J., Muller, C. L., Joe, P., Skofronick-Jackson, G., \& Kirshbaum, D. (2016). So, how much of the Earth's surface is covered by rain gauge? Bulletin of the American Meteorological Society, 98, 6978. http://dx.doi.org/10.1175/BAMS-D-14-00283.1.

Kobiyama, M., Goerl, R. F., \& Monteiro, L. R. (2018). Integração das ciências e das tecnologias para redução de desastres naturais: socio-hidrologia e socio-tecnologia. Revista Gestão \& Sustentabilidade Ambiental, 7, 206231. http://dx.doi.org/10.19177/rgsa.v7e02018206-231.

Kryvasheyeu, Y., Chen, H., Obradovich, N., Moro, E., Van Hentenryck, P., Fowler, J., \& Cebrian, M. (2016). Rapid assessment of disaster damage using social media activity. Science Advances, 2(3), 1-11. http://dx.doi.org/10.1126/sciadv.1500779.

Kullenberg, C., \& Kasperowski, D. (2016). What is citizen science?: a scientometric meta-analysis. PLoS One, 11(1), http://dx.doi.org/10.1371/journal.pone.0147152.

Lee, H., Yuan, T., Yu, H., \& Jung, A. H. (2020). Interferometric SAR for wetland hydrology: an overview of methods, challenges, and trends. IEEE Geoscience and Remote Sensing Magazine 8(1), 120-135. http://dx.doi.org/10.1109/MGRS.2019.2958653.

Lucchese, L. V., Oliveira, G. G., \& Pedrollo, O. C. (2020). Attribute selection using correlations and principal components for artificial neural networks employment for landslide susceptibility assessment. Environmental Monitoring and Assessment, 192(129), 1-22. http://dx.doi.org/10.1007/s10661-019-7968-0.

Machado, C. C. (2005). Tubarão 1974: fatos e relatos da grande enchente. Tubarão: Ed. Unisul.

McMillan, H. K., Westerberg, I. K., \& Krueger, T. (2018). Hydrological data uncertainty and its implications. WIREs. Water, 5(6), 1-14. http://dx.doi.org/10.1002/wat2.1319.

Merriam-Webster. (2020). Recuperado em 5 de outubro, de https://www.merriamwebster.com/dictionary/soft

Minayo, M. C. S. (2012). Análise qualitativa: teoria, passos e fidedignidade. Ciencia \& Saude Coletiva, 17(3), 621626.

Mondino, E., Scolobig, A., Borga, M., Albrecht, F., Mård, J., Weyrich, P., \& Di Baldassarre, G. (2020). Exploring changes in hydrogeological risk awareness and preparedness over time: a case study in northeastern Italy. Hydrological Sciences Journal, 65(7), 1049-1059. http://dx.doi.org/10.1080/02626667.2020.1729361.

Montanari, A., \& Koutsoyiannis, D. (2014). Modeling and mitigating natural hazards: stationarity is immortal! Water Resources Research, 50, 9748-9756. http://dx.doi.org/10.1002/2014WR016092.

Mostert, E. (2018). An alternative approach for socio-hydrology: case study research. Hydrology and Earth System Sciences, 22, 317-329.

Nace, R. L. (1969). Water and man: a world view (46p ). Paris: UNESCO.

NASA EarthData. (2020). Remote sensors. EARTHDATA. Recuperado em 7 de outubro de 2020, de https://earthdata.nasa.gov/learn/remote-sensors

Naulet, R. (2002). Utilisation de l'information des crues historiques pour une meilleure predetermination du risque d'inondation. Application au basin de l'Ardèche à Vallon Pont-d'Arc et St-Martin d'Ardèche (Co-tutelle de These). Sciences de la Terre et de l'Univers, Sciences de l'Eau, Université Joseph Fourier and Université du Quebec, Grenoble.

Naulet, R., Lang, M., Coeur, D., \& Gigon, C. (2001). Collaboration between historians and hydrologists on the Ardèche river (France). In T. Glade, P. Albini \& F. Frances (Eds.), The use of historical data in natural hazard assessments (pp. 113-129).

Njue, N., Stenfert Kroese, J., Gräf, J., Jacobs, S. R., Weeser, B., Breuer, L., \& Rufino, M. C. (2019). Citizen science in hydrological monitoring and ecosystem services management: state of the art and future prospects. The Science of the Total Environment, 693, 1-16. http://dx.doi.org/10.1016/j.scitotenv.2019.07.337.

Notti, D., Giordan, D., Caló, F., Pepe, A., Zucca, F., \& Galve, J. P. (2018). Potential and limitations of open satellite data for flood mapping. Yaogan Xuebao, 10(11), 1673. http://dx.doi.org/10.3390/rs10111673.

Oliveira, G. A., Brito, P. L., \& Santos, S. M. (2018). Mapeamento de Riscos Hidrológicos Utilizando Informações Geográficas Voluntárias: estudo de caso no município de Riachão do Jacuípe-Bahia. Revista Brasileira de Geografia Física, 11, 297-311. 
OpenTopography. (2020). Recuperado em 7 de outubro de 2020, de https://portal.opentopography.org/datasets

Ouarda, T. B., Rasmussen, P. F., Bobée, B., \& Bernier, J. (1998). Utilisation de l'information historique en analyse hydrologique fréquentielle. Revue des sciences de l'eau Journal of Water Science, 11, 41-49. https://doi.org/10.7202/705328ar.

Pennington, K. L., \& Cech, T. V. (2010). Introduction to water resources and environmental issues (468 p.). Cambridge: Cambridge University Press.

Rast, M., Johannessen, J., \& Mauser, W. (2014). Review of understanding of Earth's hidrological cycle: observations, theory and modelling. Surveys in Geophysics, 35(3), 491-513.

Remo, J. W. F., \& Pinter, N. (2007). Retro-modeling the Middle Mississipi River. Journal of Hydrology (Amsterdam), 337, 421-435. http://dx.doi.org/10.1016/j.jhydrol.2007.02.008.

Ribeiro Neto, A., Cirilo, J. A., Dantas, C. E. O., \& Silva, E. R. (2015). Caracterização da formação de cheias na bacia do rio Una em Pernambuco: simulação hidrológica-hidrodinâmica. Revista Brasileira de Recursos Hídricos, 20(2), 394-403.

Rocha, L. M. P. (2019). Os cientistas e a ciência cidadã: um estudo exploratório sobre a visão dos pesquisadores profissionais na experiência brasileira (Dissertação de mestrado). Instituto Brasileiro de Informação em Ciência e Tecnologia, Escola de Comunicação, Universidade Federal do Rio de Janeiro, Rio de Janeiro.

Rowley, J. (2007). The wisdom hierarchy: representations of the DIKW hierarchy. Journal of Information Science, 33(2), 163-180. http://dx.doi.org/10.1177/0165551506070706.

Ruiz-Bellet, J. L., Balasch, J. C., Tuset, J., Barriendos, M., Mazon, J., \& Pino, D. (2015). Historical, hydraulic, hydrological and meteorological reconstruction of 1874 Santa Tecla flash floods in Catalonia (NE Iberian Peninsula). Journal of Hydrology (Amsterdam), 524, 279-295. http://dx.doi.org/10.1016/j.jhydrol.2015.02.023.

Sampieri, R. H., Collado, C. F., \& Lucio, M. P. B. (2013). Metodologia de pesquisa (5. ed.). Porto Alegre, RS: Penso.

Schumann, G. J.-P., \& Domeneghetti, A. (2016). Exploiting the proliferation of current and future satellite observations of rivers. Hydrological Processes, 30, 2891-2896. http://dx.doi.org/10.1002/hyp.10825.

Schumann, G. J.-P., \& Moller, D. K. (2015). Microwave remote sensing of flood inundation. Physics and Chemistry of the Earth, 83-84, 84-95. http://dx.doi.org/10.1016/j.pce.2015.05.002.

Schumann, G., Bates, P. D., Horritt, M. S., Matgen, P., \& Pappenberger, F. (2009). Progress in integration of remote sensing-derived flood extent and stage data and hydraulic models. Reviews of Geophysics, 47(4), http://dx.doi.org/10.1029/2008RG000274

Seibert, J., \& McDonnell, J. J. (2002). On the dialog between experimentalist and modeler in catchment hydrology: use of soft data for multicriteria model calibration. Water Resources Research, 38(11), 1241. http://dx.doi.org/10.1029/2001WR000978.

Seibert, J., \& McDonnell, J. J. (2015). Gauging the ungauged basin: relative value of soft and hard data. Journal of Hydrologic Engineering, 20(1), 1-6.

Silveira, W. N., Kobiyama, M., Goerl, R. F., \& Brandenburg, B. (2009). História das Inundações em Joinville: 18512008 (153 p.). Curitiba: Ed. Organic Trading.

Sistema da Informação sobre a Biodiversidade Brasileira - SiBBr. (2020). Ciência Cidadã. Recuperado em 7 de outubro de 2020, de https://sibbr.gov.br/cienciacidada/projetos.html

Sistema Integrado de Informações sobre Desastres - S2ID. (2020). Recuperado em 5 de outubro, de https://s2id.mi.gov.br/

Sivapalan, M., Savenije, H. H. G., \& Blöschl, G. (2012). Socio-hydrology: a new science of people and water. Hydrological Processes, 26, 1270-1276.

Starkey, E., Parkin, G., Birkinshaw, S., Andy, L., Quinn, P., \& Gibson, C. (2017). Demonstrating the value of community-based ('citizen science') observations for catchment modelling and characterization. Journal of Hydrology (Amsterdam), 548, 801-817. http://dx.doi.org/10.1016/j.jhydrol.2017.03.019.

Stevaux, J. C. A. (2003). Paleoidrologia como ferramenta no gerenciamento fluvial. Boletim de Geografia UEM, 21(1), 111-114.

Sy, B., Frischknecht, C., Dao, H., Consuegra, D., \& Giuliani, G. (2020). Reconstituing past flood events: contribution of citizen science. Hydrology and Earth System Sciences, 24, 61-74. http://dx.doi.org/10.5194/hess-24-612020. 
Tarboton, D. G., Idaszak, R., Horsburgh, J. S., Heard, J., Ames, D., Goodall, J. L., Band, L., Merwade, V., Couch, A., Arrigo, J., Hooper, R., Valentine, D., \& Maidment, D. (2014). HydroShare: Advancing Collaboration through Hydrologic Data and Model Sharing. In: D. P. Ames, N. W. T. Quinn \& A. E. Rizzoli (Eds.), Proceedings of the 7th International Congress on Environmental Modelling and Software. San Diego, California, USA: International Environmental Modelling and Software Society (iEMSs). Recuperado em 7 de outubro de 2020, de http://www.iemss.org/sites/iemss2014/papers/iemss2014_submission243.pdf

Teng, J., Jakeman, A., Vaze, J., Croke, B., Dutta, D., \& Kim, S. (2017). Flood inundation modelling: a review of methods, recent advances and uncertainty analysis. Environmental Modelling \& Software, 90, 201-2016. http://dx.doi.org/10.1016/j.envsoft.2017.01.006.

Townsend, P. A. (2001). Mapping seasonal flooding in forested wetlands using multi-temporal Radarsat SAR. Photogrammetric Engineering and Remote Sensing, 67(7), 857-864.

Trend, M. G. (1978). On the reconciliation of qualitative and quantitative analyses: a case study. Human Organization, 37(4), 345-354.

União Europeia - UE. Parlamento Europeu e Conselho da União Europeia. (2007, 6 de novembro). Diretriz 2007/60/CE, de 23 de outubro de 2007, dispõe sobre avaliação e gestão dos riscos de inundações. Jornal Oficial da União Europeia, União Europeia.

United Nations International Strategy for Disaster Reduction - UNISDR. (2007). Towards a culture of prevention: disaster risk reduction begins at school good practices and lessons learned (143 p.).

Vanelli, F. M., \& Kobiyama, M. (2019). Situação atual da socio-hidrologia no mundo e no Brasil. In: Anais do $23^{\circ}$ Simpósio Brasileiro de Recursos Hídricos (SBRH). Foz do Iguaçu, PR.

Vanelli, F. M., Monteiro, L. R., Fan, F. M., \& Goldenfum, J. A. (2020). The 1974 Tubarão River flood, Brazil: hydrological and hydraulic reconstruction of the catastrophic flood. Journal of Applied Water Engineering and Research, 8(3), http://dx.doi.org/10.1080/23249676.2020.1787251.

Velásquez, N., Hoyos, C. D., Vélez, J. I., \& Zapata, E. (2020). Reconstructing the 2015 Salgar flash flood using radar retrievals and a conceptual modeling framework in an ungauged basin. Hydrology and Earth System Sciences, 24, 1367-1392. http://dx.doi.org/10.5194/hess-24-1367-2020

Vettoretti, A. (1992). História de Tubarão das origens ao século XX (426p.). Tubarão, SC: Ed. Prefeitura Municipal de Tubarão.

Vettoretti, A. (2004). Estação da piedade (169 p.). Tubarão, SC: Ed. Copiart.

Viglione, A., Di Baldassarre, G., Brandimarte, L., Kuil, L., Carr, G., Salinas, J. L., Scolobig, A., \& Blöschl, G. (2014). Insights from socio-hydrology modelling on dealing with flood risk - Roles of collective memory, risk-taking attitude and trust. Journal of Hydrology (Amsterdam), 518, 71-82.

Westerberg, I., Guerrero, J.-L., Seibert, J., Beven, K. B., \& Halldin, S. (2010). Stage-discharge uncertainty derived with a non-stationary rating curve in the Choluteca Rives, Honduras. Hydrological Processes, 25, 603-613.

Zhang, F., Zhu, X., \& Liu, D. (2014). Blending MODIS and Landsat images for urban flood mapping. International Journal of Remote Sensing, 35(9), 3237-3253. http://dx.doi.org/10.1080/01431161.2014.90335132373253.

\section{Contribuições dos autores:}

Franciele Maria Vanelli: Concepção, estruturação e redação do manuscrito.

Fernando Mainardi Fan: Apoio na estruturação, redação, fotografias da Figura 2 e revisão do manuscrito.

Masato Kobiyama: Apoio na estruturação, redação e revisão do manuscrito. 